\title{
COMENTARIO EDITORIAL
}

\section{Sildenafil: Un cambio de paradigma en el tratamiento de la disfunción sexual eréctil}

La publicación del artículo que demuestra los beneficios del sildenafil por vía oral para el tratamiento de la disfunción sexual eréctil (DSE) es un acontecimiento de gran repercusión para los pacientes con este problema y para la práctica de los médicos de atención primaria (MAP).

En el año 1990, cuando era residente de Medicina FamiLiar, leí un artículo sobre la DSE que recomendaba a todos Los MAP hacer rastreo de esta entidad en todos sus pacientes mayores de 40 años. En dicho artículo, se explicaba que el sólo hecho de que el médico hiciera la pregunta: "itiene Ud. dificultades para lograr erecciones satisfactorias?", generaba el espacio de confianza necesario para que el paciente sintiera que con su médico se "podía" hablar de "eso" (1). Y así fue que, al hacer esta pregunta, me encontré con que la prevalencia de DSE era muy alta, y me sentí reconfortado por "encontrar" dicha enfermedad mediante el rastreo. El problema es que luego no sabía qué hacer con ese hallazgo. Por eso, me parece importante hacer una suscinta reseña de este problema de salud, dando prioridad a los aspectos de la DSE que son relevantes para su manejo por parte del MAP y para lograr una correcta interacción con el especialista.

\section{Mecanismo de la erección}

Un estímulo sensorial aferente y cerebral (el más importante) envía señales que desencadenan una respuesta neurovascular que relaja el músculo liso de los cuerpos cavernosos, permitiendo así el aflujo sanguíneo que determina la erección (2) .

Para la práctica clínica cotidiana hay que recordar dos aspectos de la fisiología de la erección:

1) El estímulo sexual hace que el endotelio de los cuerpos cavernosos libere óxido nítrico. Éste estimula a la enzima guanilato ciclasa para producir GMP cíclico, que es la sustancia que relaja el músculo liso. El sildenafil actúa inhibiendo selectivamente la fosfodiesterasa que degrada el GMP cíclico, favoreciendo de esta forma el mantenimiento de la erección.

2) La adrenalina contribuye a la finalización de la erección. Este dato es muy importante para comprender lo que pasa en pacientes en quienes existe un componente de ansiedad.

\section{Enfoque del problema}

EL MAP puede resolver el $50 \%$ de las consultas por disfunciones sexuales mediante el simple reaseguro del pacien- te $y / o$ de la pareja, en cuanto a lo que es normal y lo que no es normal.

Fenómenos normales: el tiempo entre una erección y otra (periodo refractario) aumenta con la edad. Cualquier idea, preocupación, olor desagradable, dolor o temor, pueden hacer que la erección no se logre, o se pierda. Una persona, simplemente, puede no tener ganas de tener relaciones sexuales un día o durante un periodo determinado.

Fenómenos que no son normales: no es normal que un hombre, nunca, o casi nunca, logre erecciones satisfactorias que le permitan tener una relación sexual normal. Este fenómeno es independiente de la edad del paciente. Este último punto es importante ya que la erección puede lograrse en un hombre sano, incluso en edades muy avanzadas (3).

EL MAP debe explicarle al paciente que existen alternativas terapéuticas para su problema.

Debe brindar tranquilidad a él y a su pareja mostrando que, en la mayoría de los casos, lo que está ocurriendo puede ser normal.

Puede aprovecharse la ocasión para hacer entrevistas de pareja que, muchas veces, resuelven el problema. Una pareja puede no tener una buena sexualidad porque hay celos o broncas que, en ocasiones, pueden desbloquearse en pocas consultas con el MAP, sin requerir la intervención de expertos en sexología.

De las muchas causas de DSE, las que más debe tener en cuenta el MAP son las siguientes:

a) Orgánica: vascular (tanto de la macro como de la microvasculatura), neurológica, endocrinológica, postraumática o postquirúrgica, debido a drogas (las más comunes son los antihipertensivos), alcoholismo, diabetes, etc.

b) Psicógena: por problemas psicológicos 0 estrés, sin causa orgánica aparente.

c) Mixta (psicógena y orgánica) (2). Por lo general, la DSE de causa orgánica es más frecuente en pacientes mayores, se instala lentamente, el paciente tiene factores de riesgo cardiovasculares y no presenta erecciones matinales o espontáneas. La causa psicógena es más frecuente en individuos más jóvenes, sin factores de riesgo cardiovasculares, se instala bruscamente y el paciente conserva las erecciones matinales.

Existen numerosos estudios para establecer la causa de la DSE (3). Son costosos, por lo general no están cubiertos por la Obra Social o Prepaga, y los utiliza el especialista. No hay acuerdo en cuanto a su utilidad y a la necesidad de 
solicitarlos o no en la mayoría de los pacientes. Quizá el más útil de ellos sea el test de tumescencia peneana nocturna. Este test es útil para discriminar entre la DSE de causa psicógena (hay erecciones nocturnas) de la DSE de causa orgánica (no hay erecciones nocturnas).

Las opciones terapéuticas posibles antes del uso del sildenafil eran las siguientes:

a) Yohimbina: utilizada por vía oral. Si bien nunca pudo probar su eficacia en un estudio bien diseñado, probablemente tenga un efecto placebo beneficioso (4).

b) Testosterona: sólo utilizada en casos de deficiencias documentadas.

c) Terapia sexológica: de utilidad controvertida, podría servir en la DSE psicógena (3).

d) Bomba de succión por vacío: es un elemento de probada eficacia pero muy rechazado por los pacientes. Es de utilidad en todos los tipos de DSE (5).

e) Inyección intracavernosa o intrauretral de drogas vasoactivas como la papaverina, la fentolamina y/o las prostaglandinas: es el tratamiento con el que mayor experiencia se cuenta hasta el momento.

Estas drogas relajan el músculo liso facilitando la erección, independientemente del estímulo cerebral (6). Sin embargo, si éste no estimula la vía neurovascular peneana, la erección puede ser nula o muy pobre. Este tratamiento demostró su eficacia en ensayos clínicos muy bien diseñados. La aceptación es muy buena una vez que el paciente se acostumbra a inyectarse antes de la relación sexual. El problema es que muchos pacientes (y muchos médicos y esposas) lo rechazan porque no lo conocen. Es útil para todos los tipos de DSE. Su costo es elevado y, habitualmente, no está cubierto por la Obra Social o la Prepaga. Por lo general, sólo es indicado y aplicado por el especialista. Los efectos adversos clásicos de la papaverina y la fentolamina por vía intracavernosa (priapismo y fibrosis de los cuerpos cavernosos) no se presentan con el tratamiento más nuevo de inyección de una prostaglandina (alprostadil) por vía intrauretral (7). Esta última estrategia presenta como principal efecto adverso el dolor local.

Las drogas vasoactivas eran el tratamiento de elección de la DSE antes del descubrimiento del sildenafil. Las prótesis peneanas y la cirugía arterial o venosa se usan sólo cuando no hubo respuesta a los otros tratamientos.
Como puede observarse, los tratamientos no dependen, en su mayor parte, de la causa de la DSE (3). Esto genera interminables controversias. El planteo sería el siguiente: ¿vale la pena realizar estudios costosos o invasivos para determinar la "causa", si el tratamiento va a ser el mismo?

Implicancias del sildenafil como nueva herramienta terapéutica

Hemos visto que el MAP que sabe qué hacer cuando un paciente consulta por DSE tiene un importante lugar en el reaseguro, en cuanto a lo que es normal y lo que no es normal. Recordemos que puede hacer algunas entrevistas de pareja en los casos en los cuales la DSE es un síntoma de una disfunción estructural. Sin embargo, desde el punto de vista farmacológico, el MAP contaba con muy pocos recursos antes del descubrimiento del sildenafil. En resumen, el MAP que conocía las herramientas terapéuticas disponibles en ese momento sabía que, en el caso de una clara DSE de causa psicógena, si luego del reaseguro y las entrevistas de pareja, el paciente no lograba erecciones satisfactorias, debía indicársele drogas vasoactivas (además de un eventual tratamiento sexológico). Si la causa de la DSE era claramente orgánica o mixta, la intervención del MAP podía ser igualmente útil por todo lo enunciado anteriormente, pero, la necesidad de hacer un tratamiento con drogas vasoactivas era difícil de evitar y no era ético privar al paciente de este tratamiento (salvo que hubiera rechazo por parte del mismo, su pareja o su economía).

El descubrimiento del sildenafil, la publicación de este artículo (8) y la aprobación rápida de la droga pueden tener una gran repercusión para los pacientes y para los MAP. A mi juicio, el artículo publicado es metodológicamente incuestionable. Las diferencias no son sólo estadísticamente significativas sino que tienen una implicancia clínica relevante (los pacientes que utilizaron la droga tuvieron una actividad sexual mucho más satisfactoria que los que usaron placebo).

La implicancia más importante de esta publicación es que genera un "corrimiento terapéutico" del manejo de la 
DSE hacia la atención médica primaria. Recordemos que el manejo farmacológico de esta entidad era provisto casi siempre por el especialista.

También la droga genera un "corrimiento terapéutico" inevitable, y eventualmente riesgoso, desde el consultorio médico hacia la farmacia (automedicación). Esta circunstancia, aunque mal nos pese a los médicos, es muy frecuente en nuestro país, donde los controles son pobres. Sin embargo, también hay que tener en cuenta que no todos los pacientes tienen acceso a un médico con quien se sientan cómodos para hablar de la DSE. Además, el hecho de que se pueda acceder a la droga sin necesidad de consultar al médico, preserva la privacidad. Hay que mencionar también que estos pacientes han sido, y son, literalmente estafados por médicos e instituciones inescrupulosas, cuyas publicidades abundan en los diarios y revistas.

\section{¿Qué hay que saber antes de utilizar el sildenafil?}

* Para el uso de esta droga, no sería necesario conocer con certeza la causa de la DSE ya que la droga demostró ser eficaz en todos los tipos de DSE (psicógena, orgánica y mixta).

* El mecanismo de acción propuesto para el sildenafil es "fisiológico". Es decir, no provoca una erección, sino que la facilita. Esto es muy importante ya que, si un hombre se inyecta las drogas vasoactivas y luego no tiene una relación sexual, puede tener una erección o semierección. Por el contrario, en el caso del sildenafil la droga puede tomarse, y si no hay estímulo sexual, no se producirá la erección. Además, el hecho de ser administrada por vía oral una hora antes del evento, le da al hombre, y a su pareja, mayor sensación de salud y bienestar.

* En principio, el sildenafil parece ser una droga segura. Los efectos adversos fueron escasos y de poca magnitud (cefaleas y alteración en la percepción de los colores). La única contraindicación absoluta es el uso concomitante de nitritos. No debe tomarse con bebidas alcohólicas. Por otra parte, al ser una droga que fue, en un principio, probada para su uso como vasodilatador en pacientes coronarios, ha pasado por ensayos clínicos de fase 2 y 3 , lo que le da un margen de seguridad aceptable. El mecanismo de acción propuesto por el laboratorio para "vender" la droga describe sus efectos sobre los cuerpos cavernosos. Sin embargo, no sabemos si en realidad el efecto más importante se ejerce sobre el órgano principal de la erección: el cerebro. Pero más allá de estas disquisiciones, la droga parece ser segura y cumple con su objetivo clínico.

Quisiera plantear aquí un interrogante: ¿cómo puede ser que esta droga sea útil para las tres causas de DSE? ¿Existen entonces diferentes "causas" de DSE? Creo que, salvo contadas excepciones, la mayoría de las DSE son de causa mixta. $Y$ aquí es donde se hace necesario volver a la adrenalina. Para ser más claro, la mayoría de los hombres no estamos preparados para fracasar. En nuestra sociedad, la erección es una de las circunstancias en las que el hombre tiene que probar su virilidad (9). Si, por cualquier razón, ya sea un pensamiento penoso, una preocupación, un miedo, un menor flujo sanguíneo o una menor respuesta neurovascular, la erección no se logra o no se mantiene, ese hombre tendrá una herida en su narcisismo y evitará (o temerá) fracasar de nuevo. Ese miedo al fracaso es, quizás, uno de los principales determinantes de muchas disfunciones sexuales.

Por todo esto, se me ocurre que sería interesante dividir aleatoriamente en dos grupos a los tomadores de sildenafil que hayan utilizado la droga por más de seis meses con buenos resultados, dando a un grupo el sildenafily al otro grupo un placebo. Mi hipótesis es que la respuesta al placebo será mayor en este grupo que en los que utilizaron placebo en el estudio original, ya que, en los pacientes que vienen utilizando el sildenafil, podría haberse "roto" el círculo vicioso de fracaso que describí antes. Si los médicos conociéramos estos resultados, podríamos intentar "des-sildenafilinizar" al probable gran número de futuros adictos a esta droga.

\section{Dr. Esteban Rubinstein}

Unidad de Medicina Familiar y Preventiva. Hospital Italiano de Buenos Aires.

\section{Referencias}

1. Stine C, Collins M. Male Sexual Dysfunction. En Mayhew H, Selman S editors. Primary Care: Genitourinary problems in the male patient. W. B. Saunders Company; 1989 1031-1056.

2. O'Keefe M, Hunt D et al. Assesment and treatment of impotence. Med Clin North Am 79 (2) 1995.

3. Impotence. National Institute of Health Consensus Conference. JAMA; 270:83 1993.

4. Susset J, Tessier D, Wincze J, et al. Effect of Yohimbine Hydrochloride on Erectile Impotence: A double blind study. J. Urol:141:1360 1989.

5. Koreman S, Viosca S, Kaiser F et al. Use of a vacuum Tumescence Device in the Management of Impotence. J. Am. Geriatr. Soc.; 38:217 1990.

6. Grover F, Mc Clure R, Weissman R et al. Experience with Triple Drug Therapy in a Pharmacological Erection Program. J Urol; 150:1822 1993

7. Padma-Nathan H, Hellstrom W, Kaiser, F et al. NEJM; 336: 11997.

8. Goldstein I, Lue TF, H et al for The Sildenafil Study Group. Oral sildenafil in the treatment of erectile difunction. N EngL J Med 1998;338:1397-404.

9. Badinter, Elisabeth. XY, la identidad masculina. Grupo Editorial Norma, primera edición, Santa Fe de Bogotá, Colombia, 1993. 